\title{
How Far Can a Biased Random Walker Go?
}

\author{
Zhongjin Yang1, Cassidy Yang ${ }^{2}$ \\ ${ }^{1}$ Cantigny Court, Naperville, IL, USA \\ ${ }^{2}$ Division of Physics, Mathematics and Astronomy, California Institute of Technology, Pasadena, CA, USA \\ Email: yangzhongjin@gmail.com, cyyang@caltech.edu
}

Received 6 August 2015; accepted 20 September 2015; published 23 September 2015

Copyright @ 2015 by authors and Scientific Research Publishing Inc.

This work is licensed under the Creative Commons Attribution International License (CC BY).

http://creativecommons.org/licenses/by/4.0/

(c) (i) Open Access

\section{Abstract}

The random walk (RW) is a very important model in science and engineering researches. It has been studied over hundreds years. However, there are still some overlooked problems on the RW. Here, we study the mean absolute distance of an $N$-step biased random walk (BRW) in a $d$ dimensional hyper-cubic lattice. In this short paper, we report the exact results for $d=1$ and approximation formulae for $d \geq 2$.

\section{Keywords}

\section{Biased Random Walk, Monte Carlo Simulations, Stochastic Process}

\section{Introduction}

As a mathematical model, the random walk (RW) has been widely used in almost all branches of sciences and engineering [1]-[9]. Although the unbiased random walk has been studied extensively in literature, the biased random walk (BRW) has not been studied carefully in some cases.

In this short paper, we first give a brief description of the conventional results, and then report our study with some results on the BRW.

Let us consider the one dimensional BRW: a probability $p$ of going forward and a probability $(1-p)$ of going backward with uniform step length $L$. Traditionally, the average distance gone in one step is expressed as:

$$
\mu_{1}=L p+(-L)(1-p)=(2 p-1) L
$$

The variance of a one step BRW can be calculated as:

$$
\sigma_{1}^{2}=\left\langle x^{2}\right\rangle-\langle x\rangle^{2}=\left[L^{2} p+(-L)^{2}(1-p)\right]-(2 p-1)^{2} L^{2}=4 p(1-p) L^{2}
$$

After $N$ such steps, the mean distance becomes 


$$
\mu_{N}=N(2 p-1) L=2 x N L
$$

In the last expression, $x=p-\frac{1}{2}$ is used. When $p=\frac{1}{2}$ (i.e., $x=0$ ), the mean distance becomes zero. The variance of the $N$ steps is

$$
\sigma_{N}^{2}=4 p(1-p) L^{2} N=\left(1-4 x^{2}\right) N L^{2}
$$

The standard deviation of the $N$ steps is

$$
\sigma_{N}=2 \sqrt{N p(1-p)} L=\sqrt{\left(1-4 x^{2}\right) N} L
$$

In the case of the pure random walk (RW), i.e. when $p=\frac{1}{2}(x=0)$, the standard deviation of the $N$-step RW is

$$
\sigma_{N}=\sqrt{N} L .
$$

This value, known widely in literature, is usually considered as the absolute distance of an $N$-step RW. This expression is independent of the dimensions of the lattice.

However, the mean absolute distance of the $N$-step RW in a $d$-dimensional hyper-cubic lattice cannot be expressed by (6), but is the following formula [10]

$$
\left\langle\left|D_{d}(N)\right|\right\rangle=\alpha_{d}(N) \sqrt{N} L,
$$

where $\alpha_{d}(N)$ is a monotonic increasing function of dimension $d$ with saturation value of one:

$$
\begin{aligned}
& \alpha_{1}(N \rightarrow \infty)=\sqrt{2 / \pi} \approx 0.7978845608, \alpha_{2}(N \rightarrow \infty)=\sqrt{\pi} / 2 \approx 0.8862269255, \\
& \alpha_{3}(N \geq 64) \approx 0.9220, \alpha_{4}(N \geq 36) \approx 0.9410, \alpha_{5}(N \geq 18) \approx 0.9534, \quad \alpha_{6}(N \geq 13) \approx 0.9616, \cdots
\end{aligned}
$$

We compute the absolute distance for the $N$-step biased random walk (BRW). We find that (3) is a fairly good approximation for a reasonably large $N$ and $p$ away from the neighborhood of $p=\frac{1}{2}$.

In Section 2, the exact results for $d=1$ are presented. The approximation results for higher dimensions are shown in Section 3. A brief discussion is given afterward. A warning: it is possible that some of our results might have been already published in earlier literatures unknown to us.

For convenience, without loss of generality, we choose a step length of $L=1$ in hereafter expressions.

\section{Exact Results for $d=1$}

For an $N$ step biased random walker (BRW), if the walker moves forward $n$ steps with probability $p$, and moves backwards $(N-n)$ steps with probability $1-p$, this is a binomial process with probability $p$. The absolute distance from the origin will be

$$
\left|D_{1}(N, p)\right|=|N-2 n| p^{n}(1-p)^{N-n} \text {, for } N=2,3,4, \cdots .
$$

After taking the weighted configuration average, the mean absolute distance of the one-dimensional BRW can be expressed as:

$$
\left\langle\left|D_{1}(N, p)\right|\right\rangle=\sum_{n=0}^{N}|N-2 n| p^{n}(1-p)^{N-n}\left(\begin{array}{l}
N \\
n
\end{array}\right), \text { for } N=2,3,4, \cdots
$$

Using Mathematica [11], we obtain the following relationship:

$$
\left\langle\left|D_{1}(N, p)\right|\right\rangle / N=1-2 p+f(N, p) \text {, for } N=2,3,4, \cdots .
$$

where $f(N, p)$ are the polynomials of $p$ to be discussed below.

Furthermore, we obtain the following relationship (via Mathematica):

$$
f(2 m+1, p)=f(2 m, p) \text {, for } m=1,2,3, \cdots .
$$


The $f(2 m, p)$ are the $m$-term $2 m^{\prime}$ th order polynomials of $p$ with the lowest term being a $(m+1)$ 'th order term.

For convenience, we have listed some exact results for small values of $N$ as follows:

$$
\begin{gathered}
f(2, p)=2 p^{2}, \\
f(4, p)=2 p^{3}(2-p), \\
f(6, p)=2 p^{4}\left(5-6 p+2 p^{2}\right), \\
f(8, p)=2 p^{5}\left(14-28 p+20 p^{2}-5 p^{3}\right), \\
f(10, p)=2 p^{6}\left(42-120 p+135 p^{2}-70 p^{3}+14 p^{4}\right), \\
f(12, p)=2 p^{7}\left(132-495 p+770 p^{2}-616 p^{3}+252 p^{4}-42 p^{5}\right), \\
f(14, p)=2 p^{8}\left(429-2002 p+4004 p^{2}-4368 p^{3}+2730 p^{4}-924 p^{5}+132 p^{6}\right), \\
f(16, p)=2 p^{9}\left(1430-8008 p+19,656 p^{2}-27,300 p^{3}+23,100 p^{4}-11,880 p^{5}+3432 p^{6}-429 p^{7}\right), \\
f(20, p)=2 p^{11}\left(16,796-125,970 p+426,360 p^{2}-852,720 p^{3}+1,108,536 p^{4}-969,969 p^{5}\right. \\
\left.+570,570 p^{6}-217,360 p^{7}+48,620 p^{8}-4862 p^{9}\right)
\end{gathered}
$$

Further algebraic calculations yield the following recursion equations (for an even $N$, let $N=2 \mathrm{~m}$ in the following expressions):

$$
f(2 m+2, p)-f(2 m, p)=-\frac{2(2 m) !}{m !(m+1) !} p^{m+1}(1-p)^{m+1}, \text { for } m=1,2,3, \cdots
$$

i.e.

$$
f(2 m, p)=2 p^{2}-\sum_{n=2}^{m} \frac{2^{n}(2 n-3) ! !}{n !} p^{n}(1-p)^{n}, \text { for } m=2,3,4, \cdots
$$

Additionally, because $f(2 m+1, p)=f(2 m, p)$ we can obtain the following expression:

$$
\frac{\left\langle\left|D_{1}(2 m, p)\right|\right\rangle}{2 m}=\frac{\left\langle D_{1}(2 m+1, p) \mid\right\rangle}{2 m+1}=p^{2}+(1-p)^{2}-\sum_{n=2}^{m} \frac{2^{n}(2 n-3) ! !}{n !} p^{n}(1-p)^{n} \text {, for } m=2,3,4, \cdots
$$

If we let $p=\frac{1}{2}+x$ and $x \in\left[-\frac{1}{2}, \frac{1}{2}\right]$, we can see that the following is an even function for $x$ :

$$
\frac{\left\langle\left|D_{1}(2 m, x)^{*}\right|\right\rangle}{2 m}=\frac{\left\langle\left|D_{1}(2 m+1, x)^{*}\right|\right\rangle}{2 m+1}=\frac{1}{2}+2 x^{2}-\sum_{n=2}^{m} \frac{(2 n-3) ! !}{(2 n) ! !}\left(1-4 x^{2}\right)^{n} \text {, for } m=2,3,4, \cdots
$$

When $x=0$, the above becomes the unbiased random walk result [10]:

$$
\frac{\left\langle\left|D_{1}(2 m, 0)^{*}\right|\right\rangle}{2 m}=\frac{\left\langle\left|D_{1}(2 m+1,0)^{*}\right|\right\rangle}{2 m+1}=\frac{(2 m-1) ! !}{(2 m) ! !}, \text { for } m=2,3,4, \cdots
$$

In order to obtain these results, we use the following identity:

$$
\sum_{n=2}^{m} \frac{2^{n}(2 n-3) ! !}{n !}\left(\frac{1}{4}\right)^{n}+\frac{(2 m-1) ! !}{(2 m) ! !}=\sum_{n=2}^{m} \frac{(2 n-3) ! !}{(2 n) ! !}+\frac{(2 m-1) ! !}{(2 m) ! !}=\frac{1}{2}, \text { for } m=2,3,4, \cdots
$$


Therefore, Equation (15) can be expressed as a polynomial of $x^{2}$ :

$$
\begin{aligned}
\frac{\left\langle D_{1}(2 m, x)^{*} \mid\right\rangle}{2 m} & =\frac{\left\langle D_{1}(2 m+1, x)^{*} \mid\right\rangle}{2 m+1} \\
& =\frac{(2 m-1) ! !}{(2 m) ! !}+2\left(1+\sum_{n=2}^{m} \frac{(2 n-3) ! !}{(2 n-2) ! !}\right) x^{2}-\sum_{n=2}^{m} \frac{(2 n-3) ! !}{(2 n) ! !} \sum_{k=2}^{n}\left(\begin{array}{l}
n \\
k
\end{array}\right)\left(-4 x^{2}\right)^{k}, \\
& =a_{1}(N)+b_{1}(N) x^{2}-c_{1}(N) x^{4}+\cdots, \text { where } N=2 m \text { or } 2 m+1 .
\end{aligned}
$$

for $m=2,3,4, \cdots$

In order to see the quantitative behavior of the averaged absolute distance as a function of $x(=p-0.5)$, we plot Equation (18) in Figure 1 for three typical examples: $N=10$, 100, and 1000, respectively. For comparison, line $y=2 x$ is also presented in this figure. It is easy to see that the linear relationship [expressed by Equation (3)] can be used for a reasonable large $N$. Furthermore, the validity range ( $x$ value) of the linear approximation becomes larger and larger as $N$ becomes greater and greater. For reasonable accuracy, the ranges are $x>0.25,0.05$, and 0.02 , for $N=10,100$, and 1000 , respectively.

We have computed some typical values of the approximation error as follows (and partially shown in Figure 2):

$$
\left[\frac{\left\langle D_{1}(1000, x)^{*} \mid\right\rangle}{1000}-2 x\right]_{0.5 \geq x \geq 0.1}<10^{-12} \text { and }\left[\frac{\left\langle\left|D_{1}(1000, x)^{*}\right|\right\rangle}{1000}-2 x\right]_{0.5 \geq x \geq 0.15}<10^{-15} \text {. }
$$

In the range for which the linear approximation is invalid (the neighborhood of $x=0$ ), a 3-term polynomial is a fairly good approximation. In the neighborhood of $x \approx 0$, it can be expressed as

$$
\frac{\left\langle\left|D_{1}(N, x)^{*}\right|\right\rangle}{N} \approx a_{1}(N)+b_{1}(N) x^{2}-c_{1}(N) x^{4}
$$

where

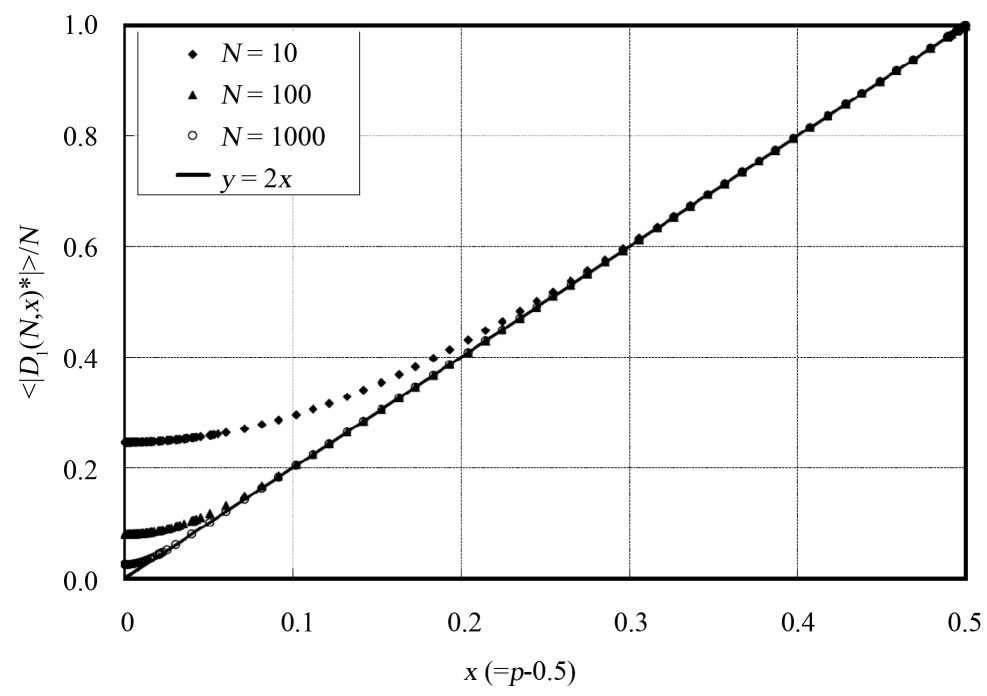

Figure 1. The normalized plot of the averaged absolute distance $\left\langle\left|D_{1}(N, x)^{*}\right|\right\rangle / N$ vs. $x\left(=p-\frac{1}{2}\right)$ for $N=10,100,1000$ in the range of $[0,0.5]$. The reference line $y=2 x$ is also shown for comparison. 


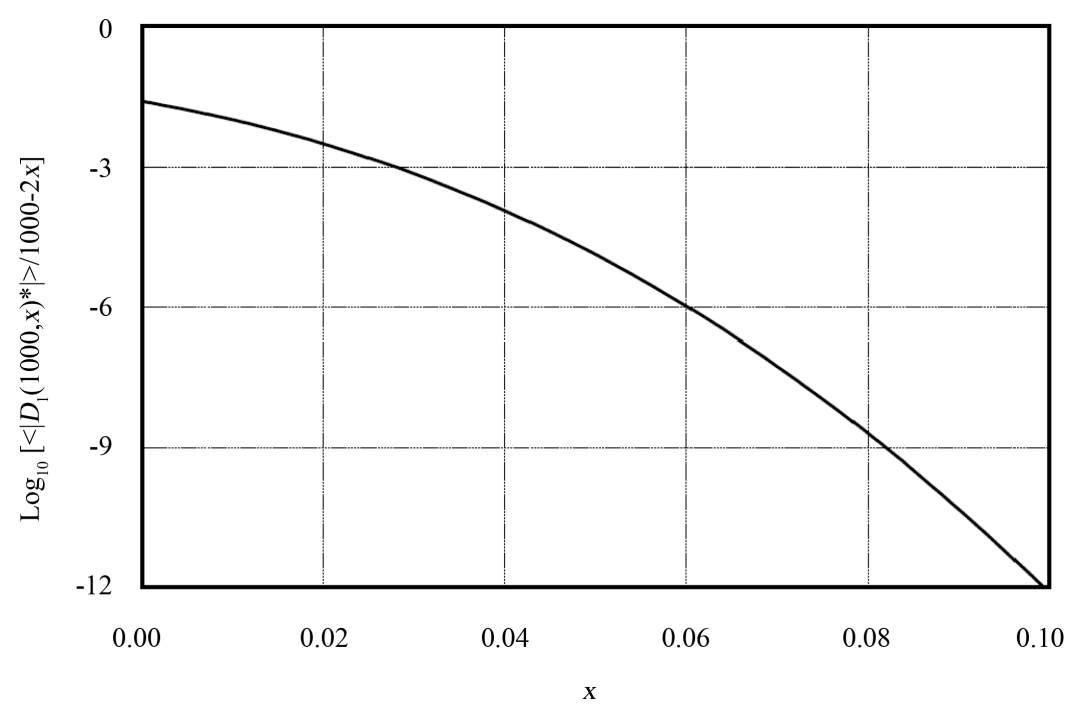

Figure 2. The semi-log plot of the difference between the normalized absolute distance and the linear approximation vs. the biased probability $x$. For accuracy reasons $\left(\sim 10^{-15}\right)$, we have only plotted the range for $x \in[0,0.1]$.

$$
\begin{gathered}
a_{1}(N)=\left\{\begin{array}{l}
\frac{(N-1) ! !}{N ! !} \text { when } N \text { is even } \\
\frac{(N-2) ! !}{(N-1) ! !} \text { when } N \text { is odd }
\end{array} \text {, for } N=2,3,4, \cdots\right. \\
b_{1}(N)=2\left(1+\sum_{n=2}^{\lfloor N / 2\rfloor} \frac{(2 n-3) ! !}{(2 n-2) ! !}\right) \text {, for } N=4,5,6, \cdots \\
c_{1}(N)=2 \sum_{n=2}^{\lfloor N / 2\rfloor} \frac{(2 n-3) ! !}{(2 n-4) ! !}, \text { for } N=4,5,6, \cdots
\end{gathered}
$$

The relationship between $a_{1}(N)$ and $a_{1}(N)$ is given by $a_{1}(N)=\alpha_{1}(N) N^{-1 / 2}$, where $a_{1}(N)$ is defined by Equation (7). The second term coefficient $b_{1}(N)$ can be expressed as $b_{1}(N)=\beta_{1}(N) \sqrt{N}$. The third term coefficient $c_{1}(N)$ can be expressed as $c_{1}(N)=\gamma_{1}(N) N^{3 / 2}$. We compute these Greek letter coefficients $a_{1}(N), \quad \beta_{1}(N)$, and $\gamma_{1}(N)$ as functions of $N$, which are shown in Figure 3 . It is easy to see that these Greek letter coefficients are of order of one. The asymptotic values of them are obtained as:

$$
\alpha_{1}(N \rightarrow \infty)=\sqrt{\frac{2}{\pi}}, \quad \beta_{1}(N \rightarrow \infty)=2 \sqrt{\frac{2}{\pi}} \text {, and } \gamma_{1}(N \rightarrow \infty)=\frac{2}{3} \sqrt{\frac{2}{\pi}}
$$

We also compute the next three terms of Equation (18): $d_{1}(N) x^{6}=\delta_{1}(N) N^{5 / 2} x^{6},-e_{1}(N) x^{8}=-\varepsilon_{1}(N) N^{7 / 2} x^{8}$ and $f_{1}(N) x^{10}=\varphi_{1}(N) N^{9 / 2} x^{10}$. The asymptotic values for the three coefficients are obtained as:

$$
\delta_{1}(N \rightarrow \infty)=\frac{4}{5} \sqrt{\frac{2}{\pi}}, \varepsilon_{1}(N \rightarrow \infty)=\frac{2}{21} \sqrt{\frac{2}{\pi}} \text {, and } \varphi_{1}(N \rightarrow \infty)=\frac{8}{135} \sqrt{\frac{2}{\pi}}
$$

From Figure 3, it is easy to see that, for a reasonable large $N$, the coefficients are very close to their asymptotic values. Therefore, in the neighborhood of $x=0$, Equation (19) can be expressed as:

$$
\frac{\left\langle\left|D_{1}(N, x)^{*}\right|\right\rangle}{N} \approx \alpha_{1}(\infty) N^{-1 / 2}+\beta_{1}(\infty) N^{1 / 2} x^{2}-\gamma_{1}(\infty) N^{3 / 2} x^{4}
$$




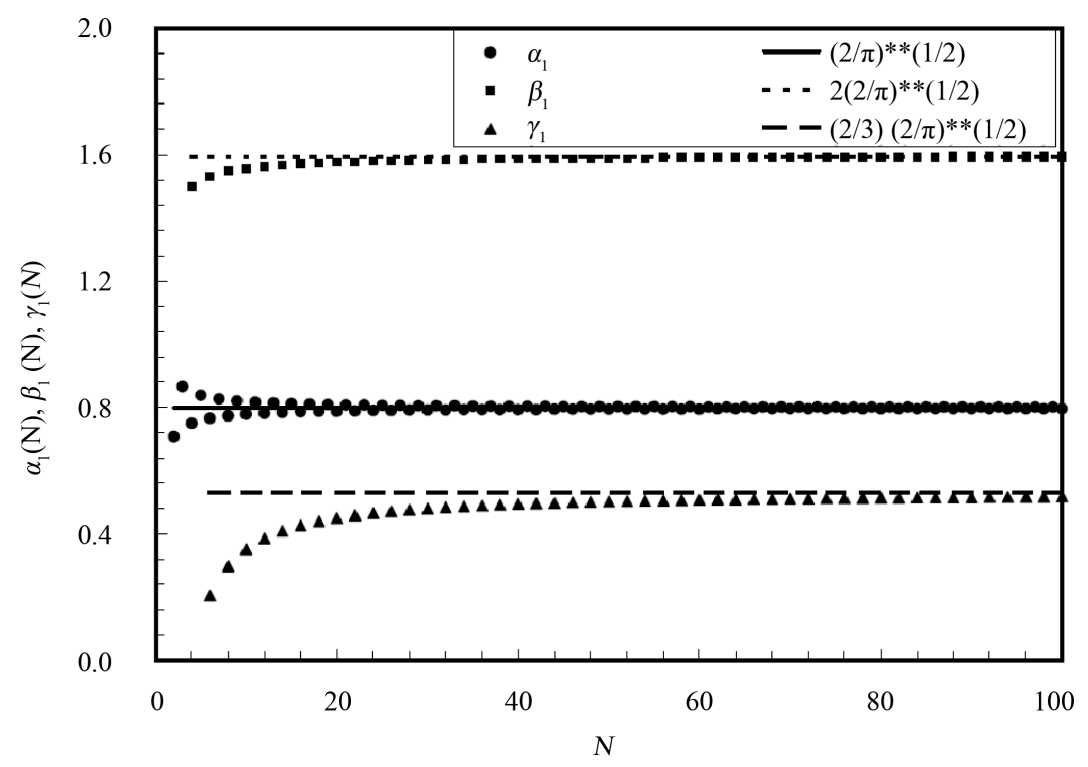

Figure 3. The coefficients, $\alpha_{1}(N), \beta_{1}(N)$, and $\gamma_{1}(N)$ as functions of the total step number $N$ for $\{N=2,3,4, \cdots, 100\}$. The asymptotic lines are also presented for comparison.

To verify the validity of the approximation (25), we plot $\left\langle\left|D_{1}(100, x)^{*}\right|\right\rangle / 100$ and $\left\langle\left|D_{1}(1000, x)^{*}\right|\right\rangle / 1000$ with exact results vs. the 3-term approximation Equation (25) for small values of $x$ in Figure 4. It is easy to see that formula (25) is a very good approximation in the neighborhood of $x \approx 0$.

\section{For $d \geq 2$}

For a $d(\geq 2)$ dimensional hyper-cubic lattice, let $p_{i}(i=1,2,3, \cdots, d)$ be the probability of walking forward along the $i$ 'th coordinate. We define $\boldsymbol{x}=\sum_{i=1}^{d} x_{i} \boldsymbol{e}_{i}$, where $x_{i}=p_{i}-\frac{1}{2} \quad(i=1,2,3, \cdots, d)$ and $\left\{\boldsymbol{e}_{i} \mid i=1,2,3, \cdots, d\right\}$ are the unit vectors. The absolute value of $|\boldsymbol{x}|$ can be expressed as: $|\boldsymbol{x}|=\sqrt{\sum_{i=1}^{d} x_{i}^{2}}$. The angle of each component is: $\cos \theta_{i}=x_{i} /|\boldsymbol{x}|(i=1,2,3, \cdots, d)$. For a reasonable large $\boldsymbol{N}$ and off the neighborhood of $|\boldsymbol{x}|=0$, this is similar to the case of $d=1$, and the displacement of the BRW should be the linear relationship, i.e.,

$$
\left\langle\left|D_{d}(N, \boldsymbol{x})^{*}\right|\right\rangle \approx 2 N \boldsymbol{x}
$$

In the neighborhood of $|\boldsymbol{x}|=0$, the three term approximation may be expressed as:

$$
\left\langle\left|D_{d}(N, \boldsymbol{x})^{*}\right|\right\rangle \approx \alpha_{d}(\infty) N^{1 / 2}+\left[\beta_{1}(\infty) N^{3 / 2} x^{2}-\gamma_{1}(\infty) N^{5 / 2} x^{4}\right] \frac{\boldsymbol{x}}{|\boldsymbol{x}|}
$$

Here, $\alpha_{d}(\infty)$ are given in Equation (8).

Alternatively, we can consider the multidimensional BRW by transposing the coordinate system so that only one direction is biased. For instance, we can consider a diffusion process via an interface in which there is a pressure applied in the direction perpendicular to the interface. In this situation, all directions except the (biased) direction perpendicular to the interface follow a pure random walk. This model can be applied to many diffusion problems in physics and chemistry. Generally speaking, we can express the dimension, $d$ as $d=g+1$, where we consider the $g$ dimensions to be unbiased random walks and the additional 1 dimension to be biased. For this model, we can study the $g$ dimensions to be unbiased random walks first. According to [10], the mean absolute 


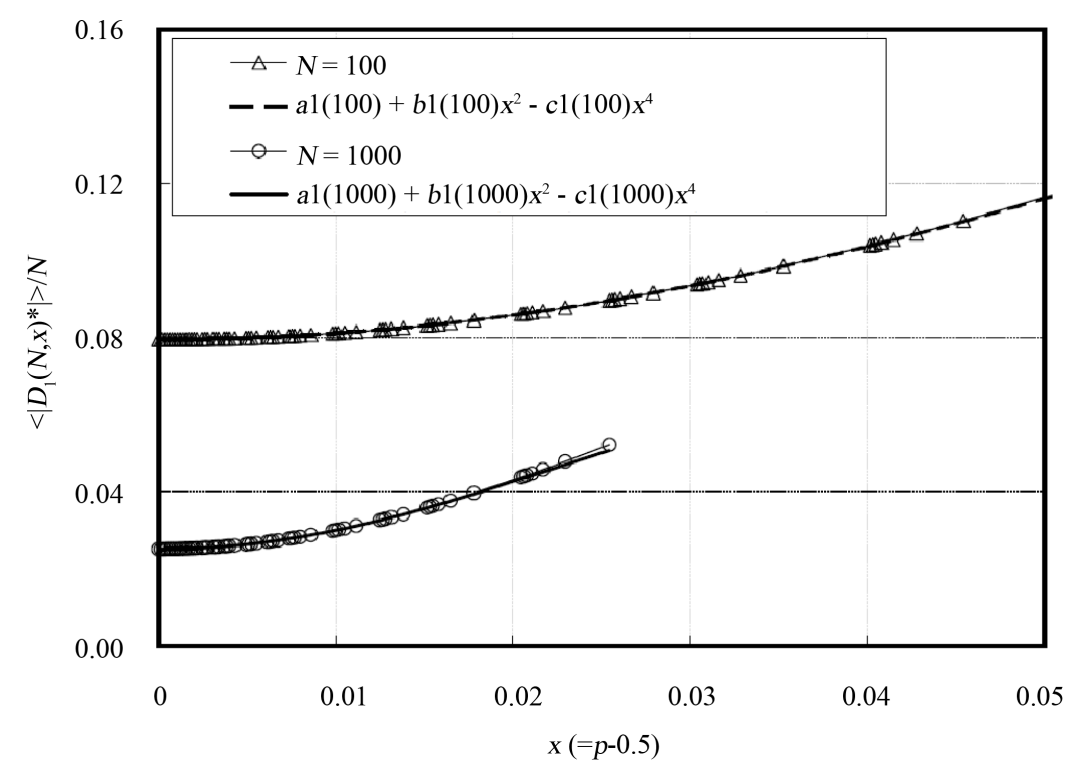

Figure 4. The normalized plot of the averaged absolute distance $\left\langle\left|D_{1}(N, x)^{*}\right|\right\rangle / N \quad$ vs. $x\left(=p-\frac{1}{2}\right)$ for $N=100$ and 1000 in the neighborhood of $x=0$. The 3-term approximations lines are also presented for comparison.

distance of the $g$-dimensional $N_{1}$-step RW

$\left\langle\left|D_{g}\left(N_{1}\right)\right|\right\rangle=\alpha_{g}\left(N_{1}\right) \sqrt{N_{1}}$, where $\alpha_{g}\left(N_{1}\right)$ is given by (8). Additionally, in a $d$-dimensional hyper-cubic lattice, the (pure random walk) displacement is perpendicular to the (biased) direction of the BRW. In many problems, we are only concerned with the absolute distance in the (biased) direction, i.e., the projection portion of the total absolute distance. For this reason, we can model it as a modified $1 d$ approach as follows: a probability $q$ walking in the $g$-dimensional hyper-cubic lattice, a probability $(1-q) p$ of going forward and a probability $(1-q)(1-p)$ of going backward in the 1-dimension. Because the $g$-dimensional lattice is perpendicular to the biased direction, the (projected) absolute distance can be expressed as:

$$
\left\langle\left|D_{d}(M, q, p)\right|\right\rangle_{1}=\sum_{N=1}^{M}\left\langle\left|D_{1}(N, p)\right|\right\rangle q^{M-N}(1-q)^{N}\left(\begin{array}{c}
M \\
N
\end{array}\right)
$$

We study two cases, one for reasonably large $p$ and the other in the neighborhood of $p \approx 0.5$, separately. For the first case, when $N$ is large enough, we have obtained the results in the previous section:

$$
\left\langle\left|D_{1}(N, p=0.5+x)\right|\right\rangle \approx 2 x N .
$$

Substituting this into (28) and using the results of the Appendix yields:

$$
\left\langle\left|D_{d}(M, q, p)\right|\right\rangle_{1} \approx \sum_{N=1}^{M} 2 x N q^{M-N}(1-q)^{N}\left(\begin{array}{c}
M \\
N
\end{array}\right)=2 x(1-q) M
$$

It is not surprising that the modification of higher dimensions on the $1 d$ result requires only multiplication of the probability factor $(1-q)$.

For the second case, i.e., in the neighborhood of $p \approx 0.5$, we can express the following:

$$
\left\langle\left|D_{d}\left(M, q, p=\frac{1}{2}+x\right)\right|\right\rangle_{1}=\sum_{N=1}^{M} q^{M-N}(1-q)^{N}\left(\begin{array}{c}
M \\
N
\end{array}\right)\left[a_{1}(N) N+b_{1}(N) N x^{2}-c_{1}(N) N x^{4}+\cdots\right]
$$

where $a_{1}(N), b_{1}(N)$ and $c_{1}(N)$ are defined by (20-22). 
Using the $1 d$ results, (31) can be estimated to be a very simple formula:

$$
\begin{aligned}
\left\langle\left|D_{d}\left(M, q, p=\frac{1}{2}+x\right)\right|_{1}\right. & \sim \sum_{N=1}^{M} q^{M-N}(1-q)^{N}\left(\begin{array}{c}
M \\
N
\end{array}\right)\left[\alpha_{1}(\infty) N^{1 / 2}+\beta_{1}(\infty) N^{3 / 2} x^{2}-\gamma_{1}(\infty) N^{5 / 2} x^{4}+\cdots\right] \\
& \sim \alpha_{1}(\infty) \sqrt{M(1-q)}+\beta_{1}(\infty)[M(1-q)]^{3 / 2} x^{2}-\gamma_{1}(\infty)[M(1-q)]^{5 / 2} x^{4}+\cdots
\end{aligned}
$$

where $\alpha_{1}(\infty), \beta_{1}(\infty)$, and $\gamma_{1}(\infty)$ are defined by (23). In the last step, (A1) in the Appendix was used.

\section{Discussion and Concluding Remarks}

The biased random walk has widely applications in various fields: for examples, a pressured diffusion process, an ionic injection with bombardment, a ballistic transport, financial market data, etc. For most natural phenomena and engineering processes, the particle number is about the order or a fraction of the Avogadro's constant $\left(\sim 10^{23}\right)$, the traditional treatment is good enough. However, the financial data and some high precision experimental data are far away from a large number, say $10^{10}$. For example in financial industry, the most active index futures, SP500, has only the order of $10^{5}$ open interest contracts before rolling the date. The daily trading volume is one or two order smaller than the open interest. Therefore, when the particle number is not large enough, one has to consider the new behavior. The present results are just the better quantitative descriptions for those phenomena. In some high precision experiments in physical sciences, one may have to measure parameters with small amount of particles. To quantify the property, the present results can provide better mathematical expressions.

\section{Acknowledgements}

The authors would like to Angel Yang for her helpful discussion.

\section{References}

[1] Feynman, R.P., Leighton, R.B. and Sands, M. (1963) Feynman Lectures on Physic. Addison-Wesley, New York, Vol. 1, 6-5, 41-9.

[2] Whitney, C.A. (1990) Random Processes in Physical Systems. Wiley, New York, 37.

[3] Uhlenbeck, G.E. and Ornstein, L.S. (1930) On the Theory of the Brownian Motion. Physical Review Letters, $36,823$. http://dx.doi.org/10.1103/PhysRev.36.823

[4] Grimmett, G. and Stirzaker, D. (2001) Probability and Random Processes. Oxford University Press, Oxford.

[5] van Kampen, N.G. (2007) Stochastic Processes in Physics and Chemistry. 3rd Edition, Elsevier, Amsterdam.

[6] Gardiner, C.W. (2004) Handbook of Stochastic Methods. 3rd Edition, Springer, Berlin. http://dx.doi.org/10.1007/978-3-662-05389-8

[7] Borodin, A.N. and Salminen, P. (2002) Handbook of Brownian Motion-Facts and Formulae. 2nd Edition, Birkhauser, Basel. http://dx.doi.org/10.1007/978-3-0348-8163-0

[8] Codling, E.A. (2003) Biased Random Walks in Biology. Ph.D. Thesis, University of Leeds, Leeds.

[9] Stauffer, D. (1985) Introduction to Percolation Theory. Talor \& Francis, London. http://dx.doi.org/10.4324/9780203211595

[10] Sun, T. and Yang, Z.J. (1992) How Far Can a Random Walker Go? Physica A: Statistical Mechanics and Its Applications, 182, 599-606. http://dx.doi.org/10.1016/0378-4371(92)90024-K

[11] Wolfram, S. (2003) Mathematica. 5th Edition, Wolfram Media, Champaign. 


\section{Appendix}

In this appendix, we present a very useful approximation formula: for a large enough $M$ and $\alpha>0$,

$$
f(\alpha)=\sum_{n=1}^{M} q^{M-n}(1-q)^{n} n^{\alpha}\left(\begin{array}{c}
M \\
n
\end{array}\right) \sim[M(1-q)]^{\alpha}
$$

It is easy to see that $f(\alpha)$ and $f^{\prime}(\alpha)$ are continuous and greater than zero, so that $f(\alpha)$ is a smooth and restricted monotonic increasing function. Let us compute some special values of $f(\alpha)$ :

$$
\begin{gathered}
f(0)=\sum_{n=0}^{M} q^{M-n}(1-q)^{n}\left(\begin{array}{c}
M \\
n
\end{array}\right)=[1+(1-q)]^{M}=1 \\
f(1)=\sum_{n=1}^{M} q^{M-n}(1-q)^{n} n\left(\begin{array}{c}
M \\
n
\end{array}\right)=\frac{\mathrm{d}}{\mathrm{d} x}\left[\sum_{n=1}^{M} q^{M-n}(1-q)^{n} x^{n}\left(\begin{array}{c}
M \\
n
\end{array}\right)\right]_{x=1}=\left.\frac{\mathrm{d}}{\mathrm{d} x}[1+(1-q) x]^{M}\right|_{x=1}=M(1-q) \\
f(2)=\sum_{n=1}^{M} q^{M-n}(1-q)^{n} n^{2}\left(\begin{array}{c}
M \\
n
\end{array}\right)=\frac{\partial}{\partial x} \frac{\partial}{\partial y}\left[\sum_{n=1}^{M} q^{M-n}(1-q)^{n}(x y)^{n}\left(\begin{array}{c}
M \\
n
\end{array}\right)\right]_{x=1, y=1} \\
=M(M-1)(1-q)^{2}+M(1-q) \sim[M(1-q)]^{2} \\
f(3)=\sum_{n=1}^{M} q^{M-n}(1-q)^{n} n^{3}\left(\begin{array}{c}
M \\
n
\end{array}\right)=\frac{\partial}{\partial x} \frac{\partial}{\partial y} \frac{\partial}{\partial z}\left[\sum_{n=1}^{M} q^{M-n}(1-q)^{n}(x y z)^{n}\left(\begin{array}{c}
M \\
n
\end{array}\right)\right]_{x=1, y=1, z=1} \\
=M(M-1)(M-2)(1-q)^{3}+3 M(M-1)(1-q)^{2}+M(1-q) \sim[M(1-q)]^{3}
\end{gathered}
$$

Numerically, we computed the values of $f\left(\frac{1}{2}\right), f\left(\frac{3}{2}\right), f\left(\frac{5}{2}\right), f\left(\frac{7}{2}\right)$, and concluded that (A1) is a very good approximation. 